\title{
Phase Diagram for Light-Induced Superconductivity in $\kappa-(\mathrm{ET})_{2}-\mathrm{X}$
}

\author{
M. Buzzi®, ${ }^{1, *}$ D. Nicoletti $\odot,{ }^{1}$ S. Fava $\odot,{ }^{1}$ G. Jotzu $\odot,{ }^{1}$ K. Miyagawa $\odot,{ }^{2}$ K. Kanoda $\odot,{ }^{2}$ A. Henderson, ${ }^{3}$ T. Siegrist, ${ }^{3}$ \\ J. A. Schlueter, ${ }^{3,4}$ M.-S. Nam, ${ }^{5}$ A. Ardavan, ${ }^{5}$ and A. Cavalleri ${ }^{1,5,}$ \\ ${ }^{1}$ Max Planck Institute for the Structure and Dynamics of Matter, 22761 Hamburg, Germany \\ ${ }^{2}$ Department of Applied Physics, University of Tokyo, 7-3-1 Hongo, Bunkyo-ku, Tokyo 113-0033, Japan \\ ${ }^{3}$ National High Magnetic Field Laboratory, 1800 E Paul Dirac Drive, Tallahassee, Florida 31310, USA \\ ${ }^{4}$ Division of Material Research, National Science Foundation, Alexandria, Virginia 22314, USA \\ ${ }^{5}$ Department of Physics, Clarendon Laboratory, University of Oxford, Oxford OX1 3PU, United Kingdom
}

(Received 27 June 2021; accepted 5 October 2021; published 2 November 2021)

\begin{abstract}
Resonant optical excitation of certain molecular vibrations in $\kappa$-(BEDT-TTF $)_{2} \mathrm{Cu}\left[\mathrm{N}(\mathrm{CN})_{2}\right] \mathrm{Br}$ has been shown to induce transient superconductinglike optical properties at temperatures far above equilibrium $T_{c}$. Here, we report experiments across the bandwidth-tuned phase diagram of this class of materials, and study the Mott insulator $\kappa$-(BEDT-TTF $)_{2} \mathrm{Cu}\left[\mathrm{N}(\mathrm{CN})_{2}\right] \mathrm{Cl}$ and the metallic compound $\kappa$-(BEDT-TTF $)_{2} \mathrm{Cu}(\mathrm{NCS})_{2}$. We find nonequilibrium photoinduced superconductivity only in $\kappa-(\mathrm{BEDT}-\mathrm{TTF})_{2} \mathrm{Cu}\left[\mathrm{N}(\mathrm{CN})_{2}\right] \mathrm{Br}$, indicating that the proximity to the Mott insulating phase and possibly the presence of preexisting superconducting fluctuations are prerequisites for this effect.
\end{abstract}

DOI: 10.1103/PhysRevLett.127.197002

Synthetic metals of the $\kappa$-(BEDT-TTF $)_{2} \mathrm{X}$ family exhibit high-temperature unconventional superconductivity [1-3] and bear some parallels with the physics of high- $T_{c}$ cuprates. In these materials, BEDT-TTF (bisethylenedithiotetrathiafulvalene, henceforth abbreviated as ET) molecules are paired in dimers and stacked in layers to form a triangular lattice [Fig. 1(a)]. Each one of the ET dimers donates an electron to the anion molecules $\mathrm{X}$, which act as a spacer layer, resulting in half-filled conduction bands. The $\kappa$-(ET) ${ }_{2} \mathrm{X}$ phase diagram [Fig. 1(b)] can be explored either by hydrostatic pressure [4,5] or by anion substitution [6]. The compound with $\mathrm{X}=\mathrm{Cu}\left[\mathrm{N}(\mathrm{CN})_{2}\right] \mathrm{Cl}(\kappa-\mathrm{Cl})$ is a Mott insulator, the one with $\mathrm{X}=\mathrm{Cu}\left[N(\mathrm{CN})_{2}\right] \mathrm{Br}(\kappa-\mathrm{Br})$, in close proximity with the Mott boundary, is a superconductor with the highest $T_{c} \approx 12 \mathrm{~K}$ for this family of materials, and the one with $\mathrm{X}=\mathrm{Cu}(\mathrm{NCS})_{2}(\kappa-\mathrm{NCS})$ is also a superconductor with slightly lower transition temperature $\left(T_{c} \approx 10 \mathrm{~K}\right)$. Because of the layered structure, the normal state optical properties resemble those of an insulator across the ET layers [7], whereas parallel to the planes the optical conductivity is insulating for the Mott phase of $\kappa-\mathrm{Cl}$ and metallic for $\kappa$ - $\mathrm{Br}$ and $\kappa$-NCS. Notably, while for $\kappa$-NCS a vortex-Nernst effect is present only in the superconducting state, close to the Mott insulating phase $(\kappa-\mathrm{Br})$ a vortexlike effect persists up to temperatures far above the

Published by the American Physical Society under the terms of the Creative Commons Attribution 4.0 International license. Further distribution of this work must maintain attribution to the author(s) and the published article's title, journal citation, and DOI. Open access publication funded by the Max Planck Society. superconducting $T_{c}$, which is suggestive of fluctuating superconductivity in the normal state $[8,9]$.

Recent work has focused on dynamical driving of $\kappa$-Br with intense laser pulses in the midinfrared [10]. Figure 2(a) summarizes the results of this experiment. Single crystals of $\kappa-\mathrm{Br}$ were cooled to temperatures for which the equilibrium response was that of a metal (here we show data taken at $30 \mathrm{~K}$ ) and driven with optical pulses tuned close to resonance with an infrared active $\mathrm{C}=\mathrm{C}$ stretching mode of the ET molecules. Their in-plane nonequilibrium optical properties were then probed with phase-sensitive $\mathrm{THz}$ time-domain spectroscopy, yielding a response reminiscent of that of a superconductor with a perfect $(R \approx 1)$ reflectivity, a gap in the real part of the optical conductivity $\sigma_{1}(\omega)$, and a $\approx 1 / \omega$ divergence in its imaginary part $\sigma_{2}(\omega)$. These superconductinglike optical features were observed for all temperatures $T \leq T^{*} \simeq 50 \mathrm{~K}$ at which the equilibrium normal state of $\kappa$ - $\mathrm{Br}$ is a highly coherent quasi-two-dimensional Fermi liquid $[11,12]$.

These experiments follow a number of qualitatively similar set of observations made in cuprates [14-17] and in fullerides [18-20]. Microscopic explanations for this class of phenomena have ranged from the transient quasistatic lattice distortions induced by nonlinear lattice vibrations [21], manipulation of competing orders [22] and the effect of dynamical modulations of the Hamiltonian parameters $[10,23]$. Recent work has highlighted the ability to cool, amplify, or otherwise manipulate normal state superconducting fluctuations [24-26]. It has been proposed [27] that a prerequisite for light-induced superconductivity should be the presence of a phase-incoherent bosonic fluid in the normal state, where superconducting fluctuations 
(a)

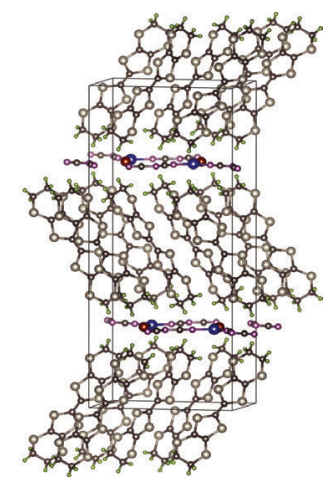

(b)

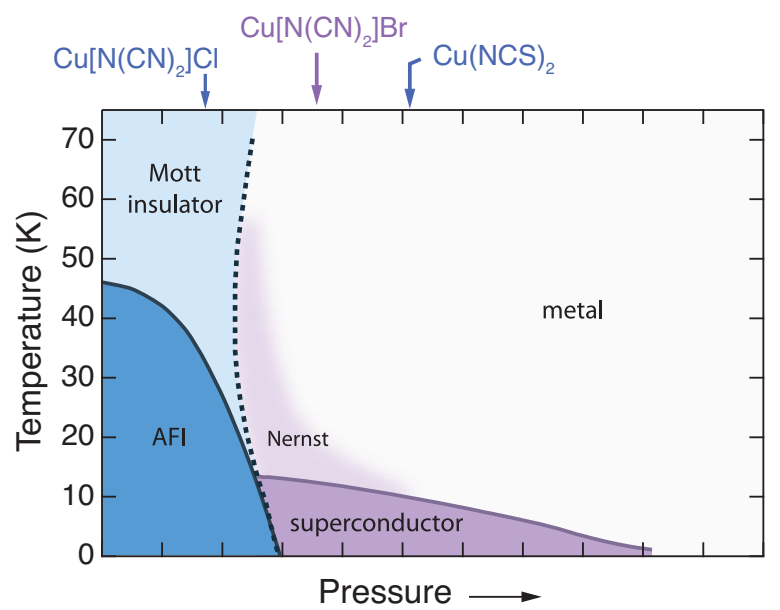

FIG. 1. (a) Crystal structure of the $\kappa$-(ET) $)_{2}$-X organic salt. (b) Temperature-effective pressure phase diagram of $\kappa$-(ET) $)_{2}-\mathrm{X}$. The three compounds studied in this work are highlighted along the horizontal axis. In proximity with the Mott insulating phase (light purple shading) measurements of the Nernst effect reveal the presence of superconducting fluctuations above $T_{c}$ [8,9].

would already be present. Here we explore this correlation by studying the response of two additional $\kappa$-(ET) ${ }_{2} \mathrm{X}$ compounds, the Mott-insulating $\kappa-\mathrm{Cl}$, and the superconducting $\kappa$-NCS, neither one exhibiting an anomalous vortexlike Nernst effect in their normal state. We find that in both materials the same vibrational excitation that was used for $\kappa$-Br does not result in a superconductinglike state.

Single crystals of $\kappa$-Cl and $\kappa$-NCS with typical dimensions of $0.5 \times 0.5 \times 0.3 \mathrm{~mm}^{3}$ were synthesized by electrocrystallization and mounted on cone-shaped holders to expose a surface that contained both the out-of-plane and one of the in-plane crystallographic directions. The crystals were photoexcited using ultrashort midinfrared pump pulses generated using an optical parametric amplifier (OPA) pumped with amplified femtosecond pulses from a Ti:sapphire laser. These pump pulses were polarized along the out-of-plane insulating crystallographic axis and tuned close to resonance with the $\nu_{27} \mathrm{C}=\mathrm{C}$ stretching mode of the ET molecules. This vibrational mode shows a strong coupling to the electronic properties, which has been theoretically shown to directly modulate the Hubbard $\mathrm{U}$ [10]. Modulation of the Hubbard $\mathrm{U}$ in time is potentially conducive to enhanced pairing in a strongly correlated material. Broadband $\mathrm{THz}$ probe pulses $(\approx 1.2$ to $7 \mathrm{THz})$ were generated in a $200-\mu \mathrm{m}$ thick $\mathrm{GaP}(110)$ crystal from the direct output of the Ti:sapphire amplifier $(800 \mathrm{~nm}$ wavelength). These $\mathrm{THz}$ probe pulses, with polarization parallel to the ET layers, were then focused on the sample and detected by electro-optic sampling after reflection in a second $200-\mu \mathrm{m}$ thick GaP (110) crystal, yielding the photoinduced changes in the low-frequency complex reflection coefficient $r(\omega)$ as a function of pump-probe time delay. While the penetration depth of the midinfrared pump $(\approx 6 \mu \mathrm{m})$ was the same for all three compounds, in $\kappa-\mathrm{Cl}$ the $\mathrm{THz}$ probe penetrated deeper inside the material $(7-20 \mu \mathrm{m})$ than in $\kappa-\mathrm{Br}$ and $\kappa$-NCS $(0.2$ to $0.6 \mu \mathrm{m})$.
The optical response functions were extracted by modeling the sample as a multilayered photoexcited stack on an unperturbed bulk volume, to account for a possible pumpprobe penetration depth mismatch [19]. We note that while this procedure is strictly needed for $\kappa$ - $\mathrm{Cl}$, in the other two compounds, where the pump penetrates deeper than the probe, this analysis yields identical results to those obtained considering a homogeneously excited medium.

Figures 2(b), 2(c) illustrate the main findings of this Letter. We report spectra of the optical properties $[R(\omega)$, $\sigma_{1}(\omega)$, and $\sigma_{2}(\omega)$ ], measured at equilibrium (red filled symbols) and 1 ps after photoexcitation (blue filled symbols) for $\kappa$-Cl [Fig. 2(b)] and $\kappa$-NCS [Fig. 2(c)]. These measurements were performed at a base temperature $T=20 \mathrm{~K}$ following the same excitation protocol that was used for $\kappa$-Br [Fig. 2(a)]. The in-plane equilibrium spectra reported for $\kappa-\mathrm{Cl}$ show a low, featureless reflectivity and a vanishingly small real part of the optical conductivity $\sigma_{1}(\omega)$ both indicative of the insulating nature of this compound. $\kappa$-NCS shows, instead, a very different response, with a high reflectivity $(R \geq 0.9)$ and a broad Drude absorption in the optical conductivity, indicative of a metallic ground state. After photoexcitation, $\kappa-\mathrm{Cl}$ displays a slight, mostly frequency independent, enhancement in both reflectivity and optical conductivity. A more pronounced effect is seen in $\kappa$-NCS where photoexcitation at this temperature induces a reduction of the reflectivity, as well as a suppression and broadening of both $\sigma_{1}(\omega)$ and $\sigma_{2}(\omega)$. No signatures of light-induced superconductivity could be observed at $T=20 \mathrm{~K}$ for both $\kappa$-Cl and $\kappa$-NCS, and all optical spectra (at equilibrium and after photoexcitation) could be captured by a Drude-Lorentz model with a finite scattering rate (red and blue solid lines, respectively).

A more complete view on the effect of photoexcitation in the $\kappa$-(ET) ${ }_{2} \mathrm{X}$ compounds is offered by Fig. 3, where we report the real part of the optical conductivity, $\sigma_{1}(\omega)$, 

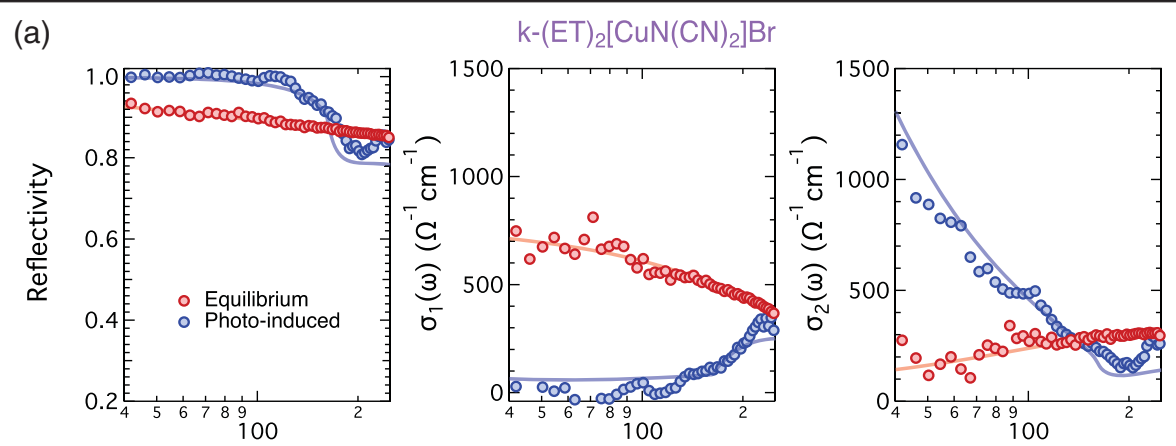

(b)
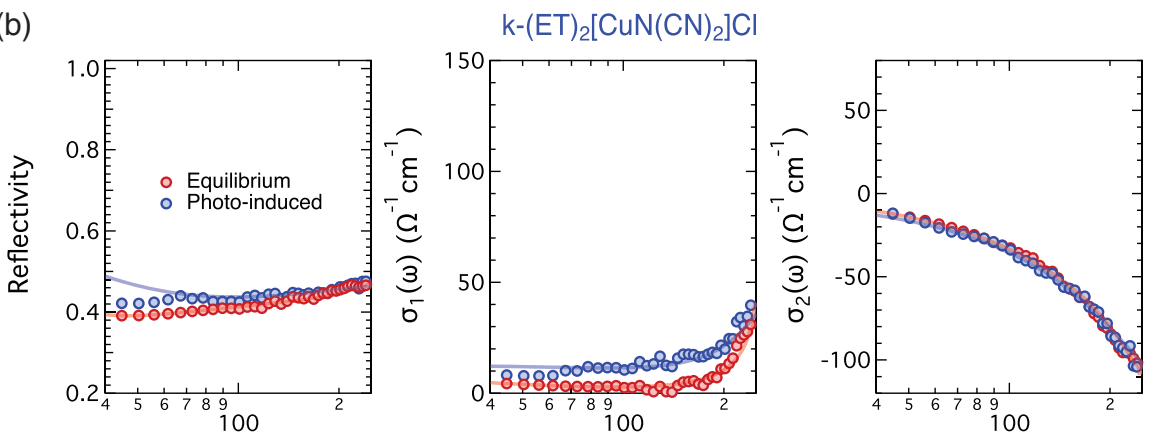

(c)
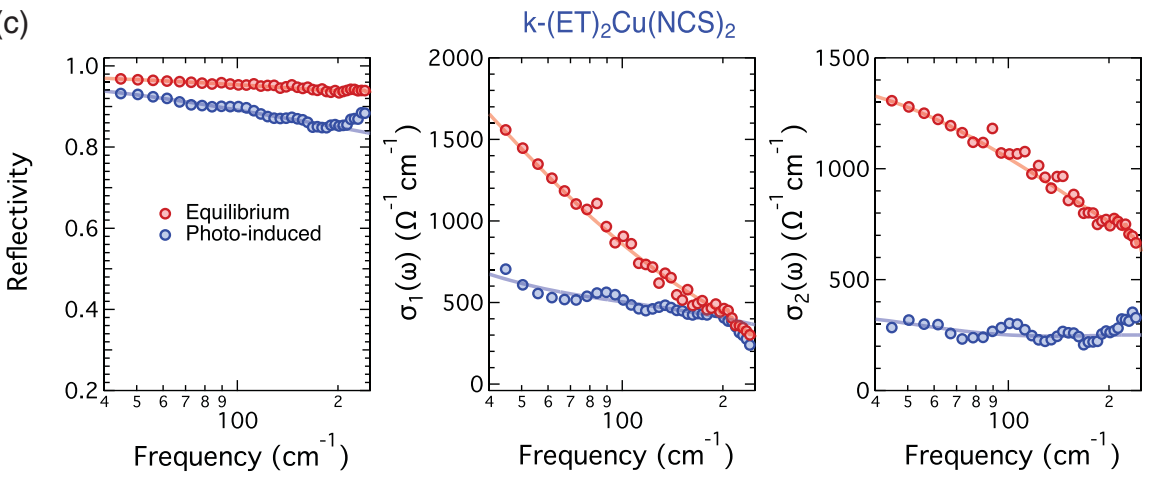

FIG. 2. (a) In-plane reflectivity, real, and imaginary part of the optical conductivity measured in $\kappa$ - $\mathrm{Br}$ at equilibrium (red circles) and at $\tau \simeq 1 \mathrm{ps}$ time delay after excitation (blue circles), at $T=30 \mathrm{~K}$. Solid lines are fits to the data with a Drude-Lorentz model (red) or a Mattis-Bardeen model for superconductors (blue). These data are reproduced from Ref. [10]. (b) Same quantities as in (a) measured in $\kappa-\mathrm{Cl}$ at equilibrium (red circles) and at $\tau \approx 1 \mathrm{ps}$ after excitation (blue circles), at $T=20 \mathrm{~K}$. Solid lines are fits to the optical spectra, which were performed with a Drude-Lorentz model for both the equilibrium and transient response. The equilibrium data are reproduced from Ref. [13]. (c) Same quantities measured for the metallic compound $\kappa$-NCS. Here, the equilibrium response was determined on the same crystal via Fourier-transform infrared spectroscopy. All data have been taken upon vibrational excitation close to resonance with the $\nu_{27} \mathrm{C}=\mathrm{C}$ stretching mode with a pump fluence of $\approx 3 \mathrm{~mJ} / \mathrm{cm}^{2}$

measured at three different temperatures in $\kappa-\mathrm{Cl}, \kappa-\mathrm{Br}$, and $\kappa$-NCS under similar excitation conditions. For $\kappa$-Br, we observe that for all temperatures $T \leq T^{*} \approx 50 \mathrm{~K}$ a clear superconductinglike gap opens at low frequencies, becoming progressively larger with decreasing temperature. This effect has been interpreted in terms of the onset of a photoinduced superconducting response [10] and fitted with an extension of the Mattis-Bardeen model for superconductors. At $T=70 \mathrm{~K}$ the response is qualitatively different: rather than the opening of a superconductinglike gap one observes an increase in $\sigma_{1}(\omega)$, indicative of enhanced metallicity.

In the case of insulating $\kappa$ - $\mathrm{Cl}$, we measure at all temperatures a slight increase of spectral weight, that one may attribute to photogeneration of free carriers. For metallic $\kappa$-NCS instead, at all $T \geq 50 \mathrm{~K}$ we observe an increase in $\sigma_{1}(\omega)$, similar to that found in $\kappa$ - $\mathrm{Br}$ for $T \geq 70 \mathrm{~K}$, thus indicative of enhanced metallicity. The photoinduced changes are different instead at $T=20 \mathrm{~K}$, where we observe a suppression in $\sigma_{1}(\omega)$ and a broadening of the Drude peak, possibly related to carrier heating. Hence, for both $\kappa-\mathrm{Cl}$ and $\kappa$-NCS the transient optical properties at all measured temperatures do not show any superconductinglike features and can be fully captured by the same Drude-Lorentz model used for the equilibrium optical spectra, with slightly varied plasma frequencies and scattering rates. 


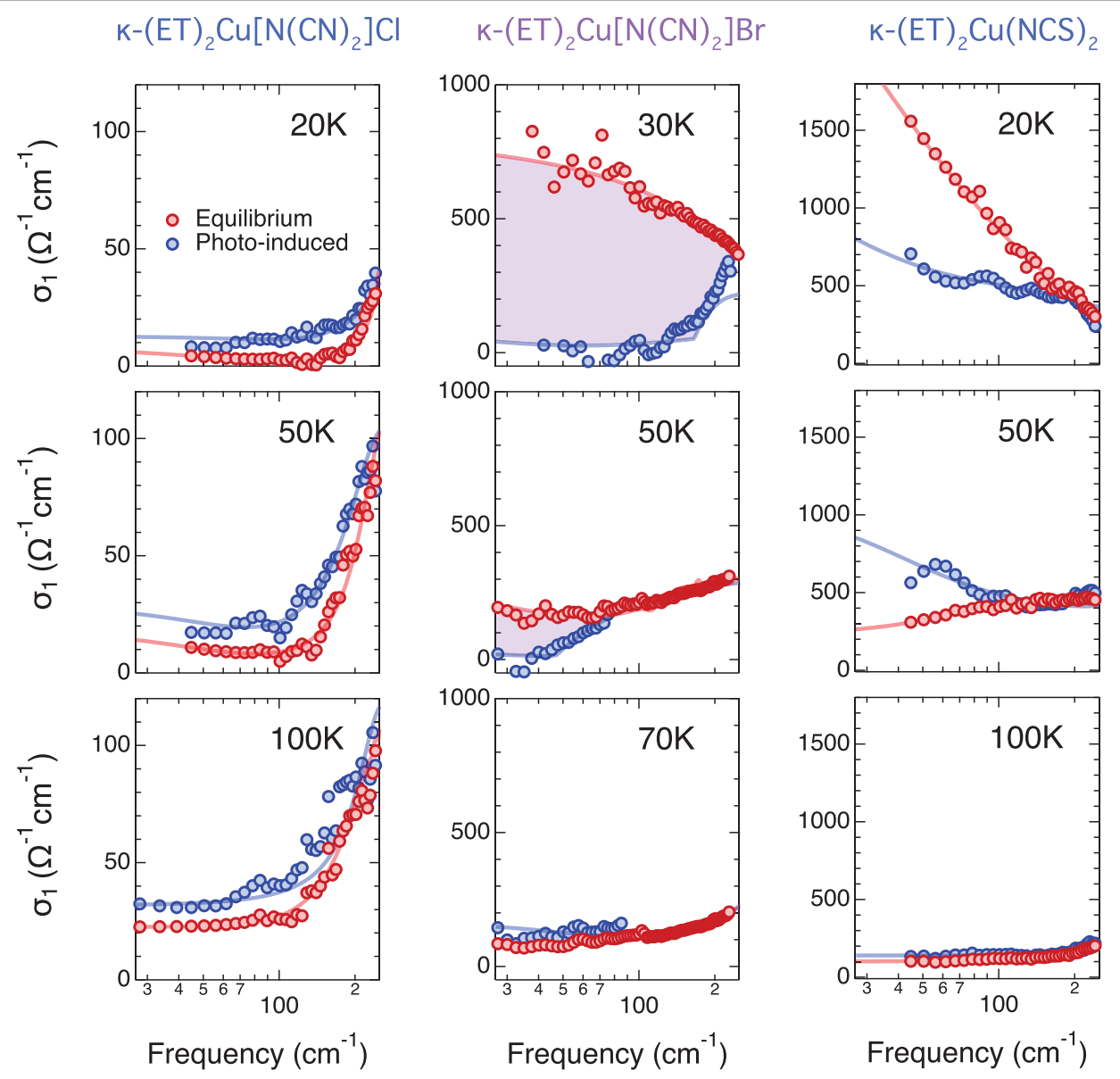

FIG. 3. (Left column) Real part of the optical conductivity measured in $\kappa$-Cl at equilibrium (red circles) and at $\tau \simeq 1 \mathrm{ps}$ after vibrational excitation (blue circles), at temperatures between 20 and $100 \mathrm{~K}$. The solid lines are fits to the optical spectra with a Drude-Lorentz model for both the equilibrium and out-of-equilibrium response. (Center column) Same quantity as in the left column, measured in $\kappa-\mathrm{Br}$ at temperatures between 30 and $70 \mathrm{~K}$. Shaded areas indicate the lost spectral weight as a superconductinglike gap appears after optical excitation. Here, a Mattis-Bardeen model for superconductors was used for the out-of-equilibrium response at temperatures $T \leq 50 \mathrm{~K}$. These data are reproduced from Ref. [10]. (right column) Same quantities measured in $\kappa$-NCS at temperatures between 20 and $100 \mathrm{~K}$. The optical spectra were all modeled with a Drude-Lorentz fit (solid lines). All data were taken at a pump fluence of $\approx 3 \mathrm{~mJ} / \mathrm{cm}^{2}$

By way of a summary, in Fig. 4(a) we report for all three samples the temperature dependence of the quantity $\sigma_{0}=\lim _{\omega \rightarrow 0} \sigma_{1}(\omega)$, i.e., the extrapolated "zero-frequency" conductivity extracted from Drude-Lorentz fits to the transient (blue) and equilibrium (red) spectra (data in Figs. 2-3). Before photoexcitation (red), $\kappa$-Cl shows the typical temperature dependence expected for an insulator, where the low-frequency conductivity increases with increasing temperature due to thermally activated carriers. In $\kappa$ - $\mathrm{Br}$ and $\kappa$-NCS the behavior is instead opposite, as expected in a metal. Notably, while in $\kappa$-Cl and $\kappa$-NCS $\sigma_{0}$ remains finite at all temperatures, in $\kappa-\mathrm{Br}$ at $T \leq T^{*} \approx 50 \mathrm{~K}$ photoexcitation causes $\sigma_{0}$ to diverge, compatible with the onset of dissipationless transport. In Fig. 4(b) we report the time dependence of the same quantity, $\sigma_{0}$, measured at $T=50 \mathrm{~K}$. In all three compounds, this changes promptly upon photoexcitation and relaxes over a few picoseconds, a timescale that is likely related to the lifetime of the driven vibrational mode. Finally, Fig. 4(c) shows the pump fluence dependent response: while in $\kappa-\mathrm{Br}$, for all fluences $F \geq 2 \mathrm{~mJ} / \mathrm{cm}^{2}, \sigma_{0}$ diverges to values compatible with a perfect conductivity, in $\kappa-\mathrm{Cl}$ and $\kappa$-NCS it always remains finite, with a significantly smoother dependence. Our observations indicate that the presence of superconducting fluctuations in the normal state and proximity to a Mott insulating state correlate to the appearance of a photoinduced state with superconductinglike optical properties. These findings are also broadly compatible with the model we put forward previously [10,28], where a periodic modulation of the Hubbard interaction parameters yielded long-range doublon correlations that may result in the generation of $\eta$ pairs and are expected to appear only below a certain value of the vertical hopping integral. One can speculate that this boundary may lay between $\kappa$ - $\mathrm{Br}$ and 

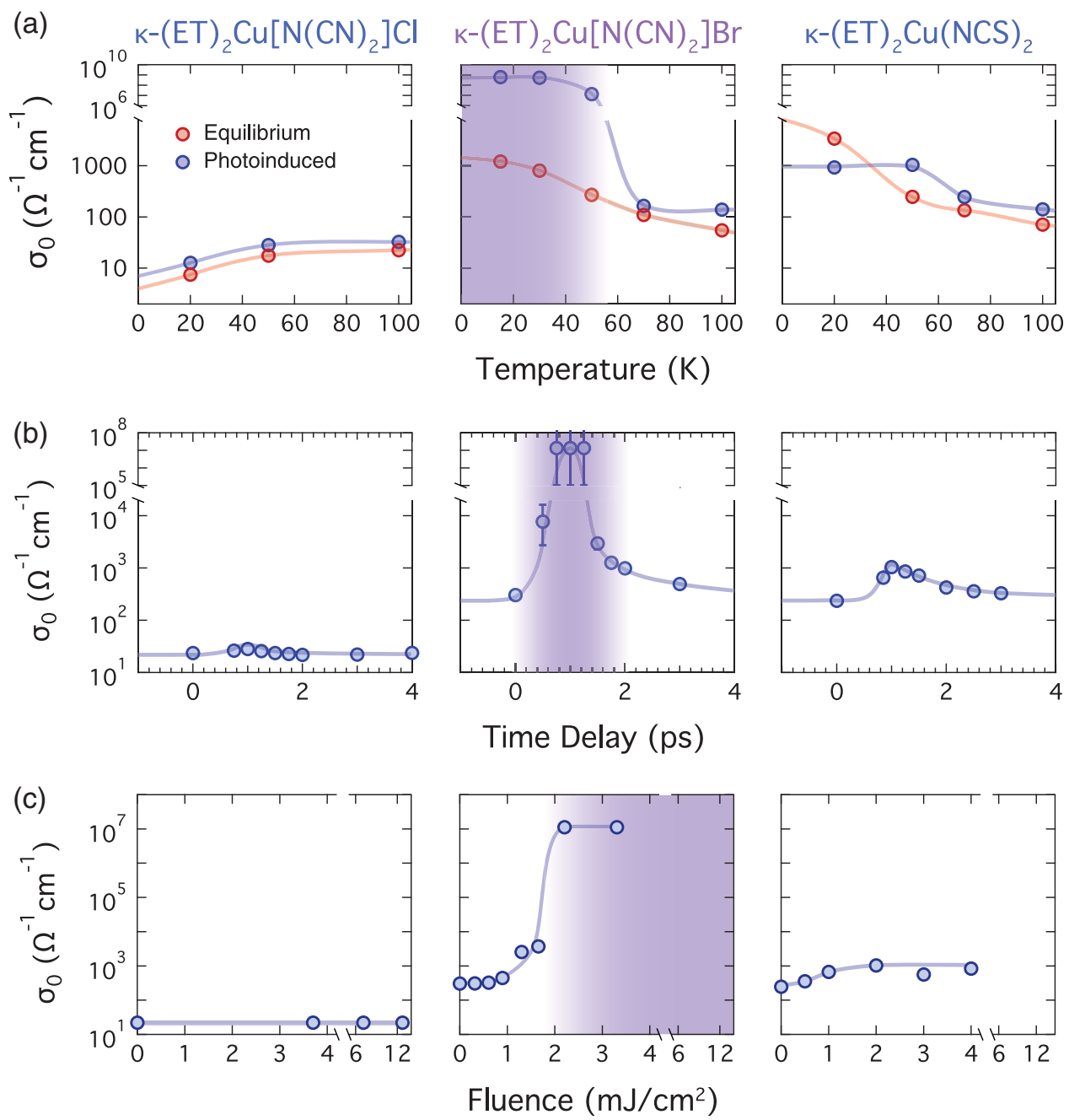

Fluence $\left(\mathrm{mJ} / \mathrm{cm}^{2}\right)$

FIG. 4. (a) Temperature dependence of $\sigma_{0}=\left.\sigma_{1}\right|_{\omega \rightarrow 0}$ (see text) in transient (blue symbols) and equilibrium (red symbols) state for $\kappa$-Cl, $\kappa$-Br, and $\kappa$-NCS. (b) Time evolution of the same quantity as in (a), measured at a base temperature $T=50 \mathrm{~K}$. Data in (a),(b) were taken upon vibration excitation at a pump fluence of $\approx 3 \mathrm{~mJ} / \mathrm{cm}^{2}$. (c) Pump fluence dependence of $\sigma_{0}$ measured at $\tau \simeq 1$ ps time delay after vibrational excitation in the same compounds. The data for $\kappa$-Br and $\kappa$-NCS were acquired at $\mathrm{T}=50 \mathrm{~K}$ while those for $\kappa$-Cl were taken at $20 \mathrm{~K}$. Solid lines are guides to the eye. Shaded areas indicate the regions of the parameter space where superconductinglike properties are found. The $\kappa$-Br data are reproduced from Ref. [10].

$\kappa$-NCS. Another recent theoretical proposal [29] discusses the appearance of superconductinglike optical properties after photoexcitation as a result of a nonequilibrium bosonic condensation of doubly occupied states, causing the simultaneous opening of a charge gap. Although within this scenario one would expect a superconductinglike response also in the insulating compound, which is not in direct agreement with our observation, this alternative interpretation highlights the importance of the vicinity to the Mott-insulating state. Note also that other measurements performed in $\mathrm{K}_{3} \mathrm{C}_{60}$ under pressure underscore the notion that the light induced superconductinglike response correlates with the electronic bandwidth of the material, becoming stronger as one approaches the Mott insulator [19].
The research leading to these results received funding from the European Research Council under the European Union's Seventh Framework Programme (FP7/20072013)/ERC Grant Agreement No. 319286 (QMAC). We acknowledge support from the Deutsche Forschungsgemeinschaft (DFG, German Research Foundation) via the excellence cluster "The Hamburg Centre for Ultrafast Imaging" (EXC 1074-project ID 194651731) and the priority program SFB925 (Project ID 170620586). J.A. Schlueter acknowledges support from the Independent Research/Development program while serving at the National Science Foundation. K. Miyagawa and K. Kanoda acknowledge support from the Japan Society for the Promotion of Science Grants No. 18H05225, No. 19H01846, No. 20K20894, and No. 20KK0060. 
*michele.buzzi@mpsd.mpg.de

† andrea.cavalleri@mpsd.mpg.de

[1] T. Ishiguro, K. Yamaji, and G. Saito, Organic Superconductors, 2nd ed., Springer Series in Solid-State Sciences (Springer-Verlag, Berlin, Heidelberg, 1998).

[2] D. Jérome, The physics of organic superconductors, Science 252, 1509 (1991).

[3] M. Lang and J. Mueller, Organic superconductors, arXiv: cond-mat/0302157.

[4] S. Lefebvre, P. Wzietek, S. Brown, C. Bourbonnais, D. Jérome, C. Mézière, M. Fourmigué, and P. Batail, Mott Transition, Antiferromagnetism, and Unconventional Superconductivity in Layered Organic Superconductors, Phys. Rev. Lett. 85, 5420 (2000).

[5] F. Kagawa, K. Miyagawa, and K. Kanoda, Unconventional critical behaviour in a quasi-two-dimensional organic conductor, Nature (London) 436, 534 (2005).

[6] K. Kanoda, Electron correlation, metal-insulator transition and superconductivity in quasi-2D organic systems, (ET) ${ }_{2}$ X, Physica (Amsterdam) 282-287C, 299 (1997).

[7] J. J. McGuire, T. Rõõm, A. Pronin, T. Timusk, J. A. Schlueter, M. E. Kelly, and A. M. Kini, Incoherent interplane conductivity of $\kappa-(\mathrm{BEDT}-\mathrm{TTF})_{2} \mathrm{Cu}\left[\mathrm{N}(\mathrm{CN})_{2}\right] \mathrm{Br}$, Phys. Rev. B 64, 094503 (2001).

[8] M.-S. Nam, A. Ardavan, S. J. Blundell, and J. A. Schlueter, Fluctuating superconductivity in organic molecular metals close to the Mott transition, Nature (London) 449, 584 (2007).

[9] M.-S. Nam, C. Mézière, P. Batail, L. Zorina, S. Simonov, and A. Ardavan, Superconducting fluctuations in organic molecular metals enhanced by mott criticality, Sci. Rep. 3, 3390 (2013).

[10] M. Buzzi et al., Photomolecular High-Temperature Superconductivity, Phys. Rev. X 10, 031028 (2020).

[11] M. Dressel, M. Dumm, N. Drichko, C. Méziere, and P. Batail, Coherent particle response of two-dimensional organic conductors upon crossing the Mott transition, J. Phys. Conf. Ser. 200, 012029 (2010).

[12] M. Dumm, D. Faltermeier, N. Drichko, M. Dressel, C. Mézière, and $\mathrm{P}$. Batail, Bandwidth-controlled Mott transition in $\kappa$-(BEDT-TTF $)_{2} \mathrm{Cu}\left[\mathrm{N}(\mathrm{CN})_{2}\right] \mathrm{Br}_{x} \mathrm{Cl}_{1-x}$ : Optical studies of correlated carriers, Phys. Rev. B 79, 195106 (2009).

[13] D. Faltermeier, J. Barz, M. Dumm, M. Dressel, N. Drichko, B. Petrov, V. Semkin, R. Vlasova, C. Meźière, and P. Batail, Bandwidth-controlled Mott transition in $\kappa$-(BEDT-TTF $)_{2} \mathrm{Cu}\left[\mathrm{N}(\mathrm{CN})_{2}\right] \mathrm{Br}_{x} \mathrm{Cl}_{1-x}$ : Optical studies of localized charge excitations, Phys. Rev. B 76, 165113 (2007).

[14] D. Fausti, R. I. Tobey, N. Dean, S. Kaiser, A. Dienst, M. C. Hoffmann, S. Pyon, T. Takayama, H. Takagi, and A. Cavalleri, Light-induced superconductivity in a stripeordered cuprate, Science 331, 189 (2011).

[15] W. Hu, S. Kaiser, D. Nicoletti, C. R. Hunt, I. Gierz, M. C. Hoffmann, M. Le Tacon, T. Loew, B. Keimer, and A. Cavalleri, Optically enhanced coherent transport in $\mathrm{YBa}_{2} \mathrm{Cu}_{3} \mathrm{O}_{6.5}$ by ultrafast redistribution of interlayer coupling, Nat. Mater. 13, 705 (2014).

[16] D. Nicoletti, E. Casandruc, Y. Laplace, V. Khanna, C. R. Hunt, S. Kaiser, S. S. Dhesi, G. D. Gu, J. P. Hill, and A. Cavalleri, Optically induced superconductivity in striped $\mathrm{La}_{2-x} \mathrm{Ba}_{x} \mathrm{CuO}_{4}$ by polarization-selective excitation in the near infrared, Phys. Rev. B 90, 100503 (2014).
[17] K. A. Cremin, J. Zhang, C. C. Homes, G. D. Gu, Z. Sun, M. M. Fogler, A. J. Millis, D. N. Basov, and R. D. Averitt, Photoenhanced metastable c-axis electrodynamics in stripeordered cuprate $\mathrm{La}_{1.885} \mathrm{Ba}_{0.115} \mathrm{CuO}_{4}$, Proc. Natl. Acad. Sci. U.S.A. 116, 19875 (2019).

[18] M. Mitrano, A. Cantaluppi, D. Nicoletti, S. Kaiser, A. Perucchi, S. Lupi, P. Di Pietro, D. Pontiroli, M. Ricco, S. R. Clark, D. Jaksch, and A. Cavalleri, Possible light-induced superconductivity in $\mathrm{K}_{3} \mathrm{C}_{60}$ at high temperature, Nature (London) 530, 461 (2016).

[19] A. Cantaluppi, M. Buzzi, G. Jotzu, D. Nicoletti, M. Mitrano, D. Pontiroli, M. Riccò, A. Perucchi, P. Di Pietro, and A. Cavalleri, Pressure tuning of light-induced superconductivity in $\mathrm{K}_{3} \mathrm{C}_{60}$, Nat. Phys. 14, 837 (2018).

[20] M. Budden, T. Gebert, M. Buzzi, G. Jotzu, E. Wang, T. Matsuyama, G. Meier, Y. Laplace, D. Pontiroli, M. Riccò, F. Schlawin, D. Jaksch, and A. Cavalleri, Evidence for metastable photo-induced superconductivity in $\mathrm{K}_{3} \mathrm{C}_{60}$, Nat. Phys. 17, 611 (2021).

[21] R. Mankowsky, A. Subedi, M. Först, S. O. Mariager, M. Chollet, H. T. Lemke, J. S. Robinson, J. M. Glownia, M. P. Minitti, A. Frano, M. Fechner, N. A. Spaldin, T. Loew, B. Keimer, A. Georges, and A. Cavalleri, Nonlinear lattice dynamics as a basis for enhanced superconductivity in $\mathrm{YBa}_{2} \mathrm{Cu}_{3} \mathrm{O}_{6.5}$, Nature (London) 516, 71 (2014).

[22] M. Först, R. I. Tobey, H. Bromberger, S. B. Wilkins, V. Khanna, A. D. Caviglia, Y. D. Chuang, W. S. Lee, W. F. Schlotter, J. J. Turner, M. P. Minitti, O. Krupin, Z. J. Xu, J. S. Wen, G. D. Gu, S. S. Dhesi, A. Cavalleri, and J. P. Hill, Melting of Charge Stripes in Vibrationally Driven $\mathrm{La}_{1.875} \mathrm{Ba}_{0.125} \mathrm{CuO}_{4}$ : Assessing the Respective Roles of Electronic and Lattice Order in Frustrated Superconductors, Phys. Rev. Lett. 112, 157002 (2014).

[23] R. Singla, G. Cotugno, S. Kaiser, M. Först, M. Mitrano, H. Liu, A. Cartella, C. Manzoni, H. Okamoto, T. Hasegawa, S. Clark, D. Jaksch, and A. Cavalleri, Thz-Frequency Modulation of the Hubbard $\mathrm{u}$ in an Organic Mott Insulator, Phys. Rev. Lett. 115, 187401 (2015).

[24] S. J. Denny, S. R. Clark, Y. Laplace, A. Cavalleri, and D. Jaksch, Proposed Parametric Cooling of Bilayer Cuprate Superconductors by Terahertz Excitation, Phys. Rev. Lett. 114, 137001 (2015).

[25] A. von Hoegen, M. Fechner, M. Först, N. Taherian, E. Rowe, A. Ribak, J. Porras, B. Keimer, M. Michael, E. Demler, and A. Cavalleri, Parametrically amplified phase-incoherent superconductivity in $\mathrm{YBa}_{2} \mathrm{Cu}_{3} \mathrm{O}_{6+x}$, arXiv:1911.08284.

[26] M. H. Michael, A. von Hoegen, M. Fechner, M. Först, A. Cavalleri, and E. Demler, Parametric resonance of Josephson plasma waves: A theory for optically amplified interlayer superconductivity in $\mathrm{YBa}_{2} \mathrm{Cu}_{3} \mathrm{O}_{6+x}$, Phys. Rev. B 102, 174505 (2020).

[27] Y. J. Uemura, Dynamic superconductivity responses in photoexcited optical conductivity and nernst effect, Phys. Rev. Mater. 3, 104801 (2019)

[28] J. Tindall, F. Schlawin, M. Buzzi, D. Nicoletti, J. â. Coulthard, H. Gao, A. Cavalleri, M. â. Sentef, and D. Jaksch, Dynamical Order and Superconductivity in a Frustrated Many-Body System, Phys. Rev. Lett. 125, 137001 (2020).

[29] Z. Dai and P. A. Lee, Superconducting-like response in driven systems near the Mott transition, arXiv:2106.08354. 